\title{
Circulating miR-30d Predicts Survival in Patients with Acute Heart Failure
}

\author{
Junjie Xiao a,b Rongrong Gao ${ }^{a}$ Yihua Bei ${ }^{b}$ Qiulian Zhou ${ }^{b}$ Yanli Zhou ${ }^{a}$ \\ Haifeng Zhanga Mengchao Jin ${ }^{a}$ Siqi Wei ${ }^{a}$ Kai Wang ${ }^{a}$ Xuejuan Xu ${ }^{a}$ \\ Wenming $\mathrm{Yao}^{\mathrm{a}}$ Dongjie Xua Fang Zhou ${ }^{\mathrm{a}}$ Jingfa Jiang ${ }^{\mathrm{b}, \mathrm{c}}$ Saumya Das ${ }^{\mathrm{d}}$ Xinli $\mathrm{Li}^{\mathrm{a}}$
}

aDepartment of Cardiology, The First Affiliated Hospital of Nanjing Medical University, Nanjing bCardiac Regeneration and Ageing Lab, Experimental Center of Life Sciences, School of Life Science, Shanghai University, Shanghai, 'Department of Cardiology, Tongji Hospital, Tongji University School of Medicine, Shanghai, China; ${ }^{\mathrm{d} C a r d i o v a s c u l a r}$ Division of the Massachusetts General Hospital and Harvard Medical School, Boston, MA, USA

\section{Key Words}

Circulating microRNAs $\cdot \operatorname{miR}-30 \mathrm{~d} \cdot$ Acute heart failure $\cdot$ Survival

\begin{abstract}
Background/Aims: Identification of novel biomarkers to identify acute heart failure (AHF) patients at high risk of mortality is an area of unmet clinical need. Recently, we reported that the baseline level of circulating miR-30d was associated with left ventricular remodeling in response to cardiac resynchronization therapy in advanced chronic heart failure patients. However, the role of circulating miR-30d as a prognostic marker of survival in patients with AHF has not been explored. Methods: Patients clinically diagnosed with AHF were enrolled and followed up for 1 year. Quantitative reverse transcription polymerase chain reactions were used to determine serum miR-30d levels. The univariate logistic regression analysis and multivariate logistic regression analysis were used to determine the predictors for all-cause mortality in AHF patients. Kaplan-Meier survival analysis was used to analyze the role of miR30d in prediction of survival. Results: A total of 96 AHF patients were enrolled and followed up for 1 year. Serum miR-30d was significantly lower in AHF patients who expired in the one year follow-up period compared to those who survived. Univariate logistic regression analysis yielded 18 variables that were associated with all-cause mortality in AHF patients, while the multivariate logistic regression analysis identified 4 variables including heart rate, hemoglobin, serum sodium, and serum miR-30d level associated with mortality. ROC curve analysis showed that hemoglobin, heart rate and serum sodium displayed poor prognostic value for AHF (AUCs not higher than 0.700) compared to miR-30d level (AUC $=0.806$ ). Kaplan-Meier survival analysis confirmed that patients with higher serum miR-30d levels had significantly lower mortality $(\mathrm{P}=0.001)$. Conclusion: In conclusion, this study shows evidence for the predictive value of circulating miR-30d as 1-year all-cause mortality in AHF patients. Large multicentre studies are further needed to validate our findings and accelerate the transition to clinical utilization.




\section{Introduction}

Acute heart failure (AHF) constitutes a leading public health burden in both developed and developing countries [1-3]. In the global context, AHF is responsible for over 1,100,000 admissions and 60,000 deaths annually $[4,5]$. AHF is a major cause of hospital admission in patients older than 65 years old, and is associated with increased morbidity and mortality $[5,6]$. Despite utilization of guideline-mandated therapy, clinical outcomes of AHF patients after hospitalization remain poor [2]. Identification of novel prognostic biomarkers will assist in tailoring of appropriate therapy for at-risk AHF patients $[1,2,7]$.

MicroRNAs (miRNAs, miRs) are a class of endogenous, non-coding RNAs of 19-25 nucleotides in length $[8,9]$. As central regulators of gene networks, miRNAs participate in several biological processes including proliferation, hypertrophy, inflammation, apoptosis and stress response [10-13]. Deregulation of miRNAs play a critical role in many cardiovascular diseases including heart failure [14-16]. Interestingly, miRNAs are present in the systemic circulation in a stable form, and the profile of plasma miRNAs can reflect cardiovascular disease [17-21]. A circulating miRNA signature for AHF patients has been reported, and more recently, the admission level of one of these AHF-associated miRNA miR423-5p, has been shown to predict 1-year mortality in AHF patients [22, 23].

We have recently described that baseline levels of circulating miR-30d in patients with advanced chronic HF, was associated with left ventricular remodeling in response to cardiac resynchronization therapy (CRT) [24]. Interestingly, miR-30d appears to play a functional role in remodeling, activating protective signaling pathways associated with adaptive hypertrophy of primary cardiomyocytes and could protect against tumor necrosis factor- $\alpha$ (TNF- $\alpha$ )-induced cardiomyocytes apoptosis [24]. However, if levels of circulating miR-30d could translate to meaningful clinical outcomes in patients with AHF is unclear.

Here a total of 96 patients with clinically diagnosed AHF were enrolled in this study and followed for up to 1 year. Serum miR-30d levels were determined by quantitative reverse transcription polymerase chain reactions (qRT-PCRs) and correlated to clinical outcomes. Our results suggest that levels of serum miR-30d in AHF correlated with mortality at 1 year even after adjustment for important clinical variables in multivariate logistic models. Collectively, this study suggests a prognostic role for circulating miR-30d in predicting 1-year mortality in AHF patients. Large multicentre investigations to validate these findings may expedite the clinical utilization of circulating miR-30d to identify at-risk AHF patients.

\section{Materials and Methods}

\section{Study cohort}

All human investigations conformed to the principles outlined in the Declaration of Helsinki and were approved by the institutional review committees of Nanjing Medical University. The AHF cohort used in this study consisted of 96 patients (60 male and 36 female) admitted to the cardiac care unit between March 2012 and April 2015.

The diagnosis of AHF was confirmed by two senior physicians in the Department of Cardiology based on the clinical and biological parameters (including N-terminal pro-B-type Natriuretic Peptide (NT-proBNP)) according to the guidelines of American Heart Association [25, 26], and defined as a rapid or gradual worsening of the signs and syptoms of heart failure. Eligible patients were those hospitalized with newonset or worsening preexisting heart failure as the primary cause of admission. The exclusion criteria are as follows: 1) Patients diagnosed with malignant tumors, cognitive dysfunction or dementia, severe mental illness, or other uncontrolled systemic disease. 2) Patients had a history of PTE/DVT, or anti-coagulation therapy within 3 months. All participants gave written informed consent before enrollment in the study. This study was registered at http://www.chictr. org/cn/. (ChiCTR-ONC-12001944).

Follow-up and endpoints

Typical clinical follow-up was performed in the outpatient setting during monthly visits or via telephone interview. The endpoints of the study were defined as all-cause mortality within 1 year from 


\section{Cellular Physiology Cell Physiol Biochem 2017;41:865-874

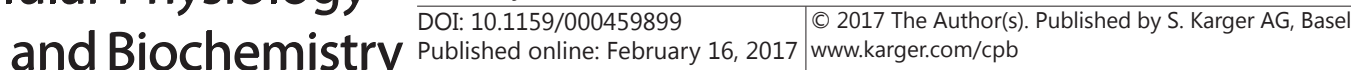 \\ Xiao et. al.: Serum miR-30d Predicts Survival in AHF}

the time they were diagnosed with AHF. During the 1-year follow-up, all enrolled AHF patients received standard treatment and management. Information concerning the death was obtained from the hospital medical record, the patient's physician, or family members.

Measurement of circulating miRNAs

Venous blood was collected in serum tubes and after a two-step centrifugation $\left(4^{\circ} \mathrm{C}\right.$ at $820 \times g$ for $10 \mathrm{~min}$, then $4^{\circ} \mathrm{C}$ at $16000 \times \mathrm{g}$ for $10 \mathrm{~min}$ ), the supernatant was transferred to RNase/DNase-free tubes and stored at $-80^{\circ} \mathrm{C}$. Total RNA was isolated from the serum samples using the mirVana PARIS isolation kit (Ambion, Austin, Texas) according to the manufacturer's instructions for serum samples without enrichment for small RNAs. Caenorhabditis elegans miR-39 (cel-miR-39) of 50 pmol/L was added as the spike-in control after the equal volume of denaturing solution was added. The Bulge-LoopTM miRNA qPCR Primer Set (RiboBio) was used to determine the expression levels of miRNAs by qRT-PCRs with Takara SYBR Premix Ex Taq ${ }^{\mathrm{TM}}$ (TliRNaseH Plus) in ABI-7900 Real-Time PCR Detection System. Cel-miR-39 was used as an internal control. The relative expression level was calculated using the $2^{-\Delta \Delta \mathrm{Ct}}$ method.

\section{Statistical analysis}

Statistical Package for the Social Sciences (SPSS, version 20.0, Chicago, IL) was used in all statistical analysis. Quantitative variables in normal distribution were expressed as mean \pm SD, and categorical variables as count (percentage). miR-30d was dichotomized into 2 categories with categorical analysis including higher than median and lower than median. Groups were compared using two independent sample $t$ test. To calculate the predictive value of variables including miR-30d for AHF, miR-30d relative expression values were calculated with the $\Delta \mathrm{Ct}$ method (mean Ct cel-miR-39-Ct target miRNA) and logtransformed by taking the base 2 logarithm, and logistic regression models using forward stepwise were constructed. Unordered categorical variables were analyzed by applying dummy variables, odds ratios and 95\% confidence interval (CI)'s were calculated for the parameters. The survival curve was drawn by the Kaplan-Meier method and survival analysis used the log-rank test. Receiver-operator characteristic (ROC) curve was established to calculate the area under the curve (AUC) of miR-30d level for diagnosing AHF patients. MedCalc software (MedCalc Software, Mariakerke, Belgium) was used to compare the areas under every ROC curves. A difference of $P<0.05$ (two-sided) was considered statistically significant.

\section{Results}

\section{Baseline characteristics}

A total of 96 patients with clinically diagnosed AHF were enrolled in this study and followed for up to 1 year. During the 1-year follow up, 17 patients died (17.7\%). Patients that died within 1 year after AHF had faster heart rate, lower levels of systolic blood pressure (SBP) and diastolic blood pressure (DBP), and more co-morbidities such as atrial fibrillation and congenital heart diseases (Table 1).

Serum miR-30d level and all cause of death within 1 year

Serum miR-30d levels in the AHF patients who survived was compared to the patients

who met the primary end-point of all-cause mortality in the follow-up period. The expression level of miR-30d in serum was significantly lower in those patients that expired compared to patients who survived during the 1 year follow up (Fig. 1) (average miR-30d Ct for survival: 29.83 \pm 0.16 ; average cel-miR-39 Ct for survival: 15.55 \pm 0.18 ; average miR-30d Ct for those expired: $30.63 \pm 0.60$; average cel-miR-39 Ct for those expired: $14.53 \pm 0.33$ ), indicating that serum miR-30d level may correlate with survival in AHF patients.

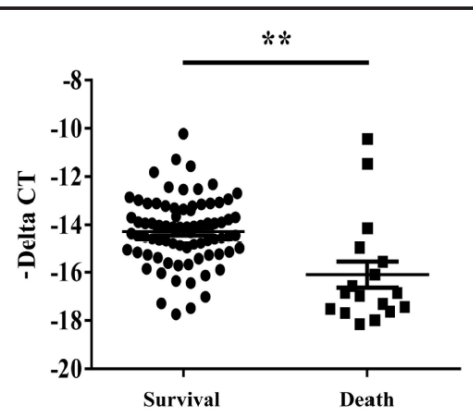

Fig. 1. Expression of serum miR-30d in acute heart failure patients survive during 1 year follow up comparing to those died. Y axis: -delta CT. ${ }^{* *}, \mathrm{P}<0.01$. 


\section{Cellular Physiology Cell Physiol Biochem 2017;41:865-874 \begin{tabular}{ll|l} 
and Biochemistry Published online: February 16, 2017 & $\begin{array}{l}\text { (c) } 2017 \text { The Author(s). Published by S. Karger AG, Basel } \\
\text { www.karger.com/cpb }\end{array}$ \\
\hline
\end{tabular} \\ Xiao et. al.: Serum miR-30d Predicts Survival in AHF}

Table 1. Acute heart failure patient characteristics with different clinical outcomes (No. or mean \pm SD)

\begin{tabular}{|c|c|c|c|c|c|c|c|}
\hline \multirow[b]{2}{*}{ Age (years) } & \multicolumn{3}{|c|}{ Survival $(n=79)$} & \multicolumn{3}{|c|}{ Death $(n=17)$} & \multirow{2}{*}{$\frac{P \text { value }}{0.919}$} \\
\hline & 61 & \pm & 16 & 62 & \pm & 17 & \\
\hline Gender (No.) & & & & & & & 0.173 \\
\hline Male & & 52 & & & 8 & & \\
\hline Female & & 27 & & & 9 & & \\
\hline Heart rate (beats/min, mean $\pm \mathrm{SD}$ ) & 78 & \pm & 17 & 89 & \pm & 16 & 0.017 \\
\hline NT-proBNP (ng/mL) & 1934.00 & \pm 2 & 2331.59 & 2847.60 & $0 \pm 2$ & 117.09 & 9.794 \\
\hline CK-MB (IU/L) & 14.73 & \pm & 9.17 & 59.76 & \pm & 229.38 & 0.287 \\
\hline ASA class (No.) & & & & & & & 0.282 \\
\hline I & & 1 & & & 0 & & \\
\hline II & & 15 & & & 2 & & \\
\hline III & & 44 & & & 7 & & \\
\hline IV & & 19 & & & 8 & & \\
\hline Ejection Fraction (\%) & 47 & \pm & 16 & 48 & \pm & 18 & 0.797 \\
\hline HFpEF(No.) & & 35 & & & 8 & & 0.986 \\
\hline SBP (mmHg, mean \pm SD) & 129 & \pm & 26 & 113 & \pm & 17 & 0.015 \\
\hline $\mathrm{DBP}(\mathrm{mmHg}$, mean $\pm \mathrm{SD})$ & 77 & \pm & 15 & 67 & \pm & 6 & 0.001 \\
\hline \multicolumn{8}{|l|}{ Co-morbidities (No. (\%)) } \\
\hline Atrial fibrillation & \multicolumn{3}{|c|}{$26(32.91 \%)$} & \multicolumn{3}{|c|}{$10(58.82 \%)$} & 0.045 \\
\hline Hypertension & \multicolumn{3}{|c|}{$41(51.90 \%)$} & \multicolumn{3}{|c|}{$6(35.29 \%)$} & 0.214 \\
\hline Acute coronary syndromes & \multicolumn{3}{|c|}{$8(10.13 \%)$} & \multicolumn{3}{|c|}{$0(0)$} & 0.343 \\
\hline Ischemic heart disease & \multicolumn{3}{|c|}{$16(20.25 \%)$} & \multicolumn{3}{|c|}{$2(11.76 \%)$} & 0.622 \\
\hline Cardiomyopathy & \multicolumn{3}{|c|}{$27(34.18 \%)$} & \multicolumn{3}{|c|}{$4(23.53 \%)$} & 0.572 \\
\hline Valvular disease of the heart & \multicolumn{3}{|c|}{$17(21.52 \%)$} & \multicolumn{3}{|c|}{$5(29.41 \%)$} & 0.701 \\
\hline Pulmonary heart disease & \multicolumn{3}{|c|}{$3(3.80 \%)$} & \multicolumn{3}{|c|}{$0(0)$} & 1.000 \\
\hline Hyperthyreosis & \multicolumn{3}{|c|}{$2(2.53 \%)$} & \multicolumn{3}{|c|}{$1(5.88 \%)$} & 1.000 \\
\hline Congenital heart diseases & \multicolumn{3}{|c|}{$4(5.06 \%)$} & \multicolumn{3}{|c|}{$4(23.53 \%)$} & 0.044 \\
\hline Diabetes mellitus & \multicolumn{3}{|c|}{$20(25.32 \%)$} & \multicolumn{3}{|c|}{$5(29.41 \%)$} & 0.965 \\
\hline Renal insufficiency & \multicolumn{3}{|c|}{$2(2.53 \%)$} & \multicolumn{3}{|c|}{$1(5.88 \%)$} & 1.000 \\
\hline
\end{tabular}

Serum miR-30d level in relation to baseline characteristics

The AHF cohort was divided into 2 groups according to the median of serum miR-30d levels. These two groups of AHF patients had similar age and gender characteristics, and received standard treatment and management for the whole course from admission to discharge. Lower incidence of atrial fibrillation and higher incidence of cardiomyopathy were found in miR-30d high level group (Table 2). Expect for these two variables, binary logistic regression showed that miR-30d was not associated with other baseline clinical characteristics (Table 3), suggesting that serum miR-30d was not a surrogate marker of other comorbidities in this cohort.

\section{Serum miR-30d as a prognostic indicator for $A H F$}

In univariate logistic regression analysis, the variable selection process yielded 18 variables that closely related to the outcomes of AHF (Table 4). Of the 18 variables, factors including heart rate, serum sodium, blood urea nitrogen, hemoglobin, cystatinc C, uric acid and serum miR-30d level were chosen as independent variables. These variables were then included in the multivariate logistic regression analysis. Our results showed that higher hemoglobin, serum sodium and miR-30d level were associated with a reduced risk of death caused in AHF patients (OR: 0.962 [0.930 - 0.995], 0.759 [0.610 to 0.944] and 0.610 [0.409 to $0.911]$, respectively, all $\mathrm{P}<0.05)$, while higher heart rate correlated with increased mortality after AHF (OR: 1.048 [1.000 - 1.098], P=0.048) (Table 5).

We further evaluated the predictive power of these 4 variables to the outcomes of AHF by ROC curve analysis. Hemoglobin, heart rate and serum sodium displayed only moderate prognostic values for AHF (AUCs $\leq 0.700)$ compared to miR-30d level $(A U C=0.806)$ (Fig. 2).Combination of two or three variables (excluding miR-30d) also did not improve the KARGER 


\section{Cellular Physiology Cell Physiol Biochem 2017;41:865-874 \begin{tabular}{ll|l} 
DOI: 10.1159/000459899 & $\begin{array}{l}\text { O } 2017 \text { The Author(s). Published by S. Karger AG, Basel } \\
\text { www.karger.com/cpb }\end{array}$ \\
\cline { 2 - 3 }
\end{tabular} \\ Xiao et. al.: Serum miR-30d Predicts Survival in AHF}

Table 2. Patient characteristics in groups with different miR-30d level (No. or mean \pm SD). NT-proBNP: $\mathrm{N}$-terminal B-type natriuretic peptide; CK-MB: creatinine kinaseisoenzyme; ASA: American society of anesthesiologists; HFpEF: heart failure preserved ejection fraction; SBP: systolic blood pressure; DBP: diastolic blood pressure

\begin{tabular}{|c|c|c|c|c|c|c|c|}
\hline & miR-30d lor & Wleve & $(n=52)$ & $\mathrm{miR}-30 \mathrm{dr}$ & & el $(n=44)$ & $P$ value \\
\hline Age (years) & 63 & \pm & 17 & 60 & \pm & 15 & 0.402 \\
\hline Gender (No.) & & & & & & & 0.290 \\
\hline Male & & 25 & & & 35 & & \\
\hline Female & & 27 & & & 9 & & \\
\hline Heart rate (beats/min, mean $\pm S D$ ) & 82 & \pm & 20 & 78 & \pm & 15 & 0.272 \\
\hline ASA class (No.) & & & & & & & 0.537 \\
\hline I & & 1 & & & 0 & & \\
\hline II & & 6 & & & 11 & & \\
\hline III & & 25 & & & 26 & & \\
\hline IV & & 12 & & & 15 & & \\
\hline Ejection Fraction (\%) & 51 & \pm & 16 & 45 & \pm & 17 & 0.126 \\
\hline HFpEF(No.) & & 21 & & & 22 & & 0.275 \\
\hline SBP $(m m H g$, mean $\pm S D)$ & 127.70 & \pm & 28.32 & 124.98 & \pm & 22.58 & 0.601 \\
\hline $\mathrm{DBP}(\mathrm{mmHg}$, mean $\pm \mathrm{SD})$ & 75.80 & \pm & 16.26 & 74.71 & \pm & 12.16 & 0.710 \\
\hline Co-morbidities (No. (\%)) & & & & & & & \\
\hline Atrial fibrillation & 23( & 44.230 & & & 29.5 & $\%)$ & 0.006 \\
\hline Hypertens & 20( & $38.46^{\circ}$ & & & 51.3 & $\%$ ) & 0.528 \\
\hline ronary syndromes & & $7.69 \%$ & & & 9.09 & & 1.000 \\
\hline Ischen & 10( & 19.23 & & & 8.18 & & 0.330 \\
\hline Cardion & & $17.31 \%$ & & & $(50$ & & 0.023 \\
\hline Valvular disease of the heart & & $3(25 \%$ & & & 0.45 & & 0.155 \\
\hline Pulmonary heart disease & & $1.92 \%$ & & & 4.55 & & 1.000 \\
\hline Hyperthyr & & $3.85 \%$ & & & 2.27 & & 0.883 \\
\hline eart diseases & & $7.69 \%$ & & & 9.09 & & 1.000 \\
\hline Diabete & & 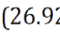 & & & $(25$ & & 0.235 \\
\hline Renal insufficiency & & $1.92 \%$ & & & 4.55 & & 1.000 \\
\hline NT-proBNP (ng/mL) & 1808.41 & \pm & 2510.08 & 2973.69 & \pm & 4773.67 & 0.135 \\
\hline D-Dimer (mg/L) & 1.10 & \pm & 3.61 & 2.05 & \pm & 3.69 & 0.223 \\
\hline Plasma potassium (mmol/L) & 3.98 & \pm & 0.64 & 3.98 & \pm & 0.56 & 0.974 \\
\hline odium $(\mathrm{mmol} / \mathrm{L})$ & 140.19 & \pm & 3.28 & 139.51 & \pm & 5.18 & 0.457 \\
\hline CK-MB (IU/L) & 14.73 & \pm & 9.17 & 59.76 & \pm & 229.38 & 0.287 \\
\hline Blood urea nitrogen $(\mathrm{mg} / \mathrm{dL})$ & 7.63 & \pm & 3.01 & 10.30 & \pm & 6.68 & 0.018 \\
\hline $\operatorname{Scr}(\mu \mathrm{mol} / \mathrm{L})$ & 87.75 & \pm & 28.83 & 105.84 & \pm & 57.82 & 0.052 \\
\hline Hemoglobin $(\mathrm{g} / \mathrm{L})$ & 135.06 & \pm & 17.33 & 126.82 & \pm & 28.19 & 0.096 \\
\hline RDW (\%) & 14.02 & \pm & 2.46 & 15.07 & \pm & 2.06 & 0.028 \\
\hline $\operatorname{ALT}(\mathrm{U} / \mathrm{L})$ & 90.47 & \pm & 290.49 & 71.12 & \pm & 171.68 & 0.702 \\
\hline $\operatorname{AST}(\mathrm{U} / \mathrm{L})$ & 135.73 & \pm & 515.48 & 84.77 & \pm & 244.20 & 0.554 \\
\hline Cystatinc C (n & 1.35 & \pm & & 1.64 & \pm & 1.01 & 0.114 \\
\hline Uric acic & 457.02 & \pm & 155.46 & 518.91 & \pm & 179.40 & 0.078 \\
\hline Ejection Fraction (\%) & 45.17 & \pm & 16.54 & 50.54 & \pm & 16.26 & 0.126 \\
\hline LVDD (mm) & 60.18 & \pm & 14.29 & 56.48 & \pm & 13.24 & 0.210 \\
\hline
\end{tabular}

Table 3. Association of miR$30 d$ serum levels with baseline characteristics. ASA: American society of anesthesiologists

\begin{tabular}{lccc}
\hline Variable & Odds ratio & $95 \%$ CI & $P$ value \\
\hline Age & 0.989 & $0.963-1.015$ & 0.398 \\
Gender & 0.565 & $0.681-3.594$ & 0.291 \\
ASA class & 0.972 & $0.549-1.722$ & 0.923 \\
Heart rate & 0.987 & $0.964-1.010$ & 0.271 \\
Pneumonia & 0.707 & $0.247-2.022$ & 0.518 \\
Atrial fibrillation & 0.304 & $0.128-0.721$ & 0.007 \\
Ischemic heart disease & 0.600 & $0.213-1.686$ & 0.333 \\
Cardiomyopathy & 2.852 & $1.141-7.128$ & 0.025 \\
Acute coronary syndromes & 0.833 & $0.196-3.546$ & 0.805 \\
Hyperthyreosis & 0.412 & $0.036-4.700$ & 0.412 \\
Hypertension & 0.998 & $0.986-1.010$ & 0.742 \\
Congenital heart diseases & 0.833 & $0.196-3.546$ & 0.805 \\
Diabetes mellitus & 0.575 & $0.229-1.442$ & 0.238 \\
Renal insufficiency & 1.720 & $0.151-19.632$ & 0.662 \\
\hline
\end{tabular}

clinically prognostic value (Fig. 3A, Fig. 3B and Fig. 4). While AUC for combination of all four variables reached 0.872 , which was significantly improved over the AUC areas of the single variables; this combination was not statistically different compared to serum miR30d ( $>0.05$ ) (Fig. 4), indicating that serum miR-30d could be a prognostic indicator for AHF. Moreover, we correlated serum miR-30d to the established conventional marker NT- 
Table 4. Prediction of death (univariate analysis). NT-proBNP: N-terminal B-type natriuretic peptide; CK-MB: creatinine kinase isoenzyme; Scr: serum creatinine; ALT: alanine aminotransferase; AST: aspartate transaminase; LVDD: left ventricular end-diastolic dimension

\begin{tabular}{lccc}
\hline Variable & Odds ratio & $95 \% \mathrm{CI}$ & $P$ value \\
\hline Age & 1.002 & $0.969-1.036$ & 0.918 \\
Gender & 0.444 & $0.154-1.286$ & 0.135 \\
NT-proBNP & 1.000 & $1.000-1.000$ & 0.569 \\
D-Dimer & 0.981 & $0.819-1.173$ & 0.830 \\
Heart rate & 1.038 & $1.005-1.072$ & 0.025 \\
Serum potassium & 1.606 & $0.662-3.899$ & 0.295 \\
Serum sodium & 0.826 & $0.724-0.941$ & 0.004 \\
CK-MB & 0.998 & $0.986-1.010$ & 0.742 \\
Blood urea nitrogen & 1.104 & $1.009-1.208$ & 0.032 \\
Scr & 1.003 & $0.992-1.013$ & 0.622 \\
Hemoglobin & 0.968 & $0.945-0.991$ & 0.007 \\
ALT & 1.003 & $1.000-1.007$ & 0.053 \\
AST & 1.003 & $0.999-1.006$ & 0.160 \\
Cystatinc C & 2.189 & $1.117-4.291$ & 0.022 \\
Uric acid & 1.003 & $1.000-1.006$ & 0.047 \\
Ejection Fraction & 1.004 & $0.972-1.037$ & 0.803 \\
LVDD & 0.967 & $0.926-1.009$ & 0.118 \\
miR-30d & 0.472 & $0.316-0.705$ & $<0.001$ \\
\hline
\end{tabular}

Table 5. Prediction of death (multivariate analysis)

\begin{tabular}{lccc}
\hline Variable & Odds ratio & $95 \% \mathrm{CI}$ & P value \\
\hline Hemoglobin & 0.962 & $0.930-0.995$ & 0.025 \\
Heart rate & 1.048 & $1.000-1.098$ & 0.049 \\
Serum sodium & 0.759 & $0.610-0.944$ & 0.049 \\
miR-30d & 0.610 & $0.409-0.911$ & 0.016 \\
\hline
\end{tabular}

Fig. 2 The receiver-operator characteristic (ROC) curves for acute heart failure patients survive during 1 year follow up comparing to those died with single variable. The receiver-operator characteristic (ROC) curves for distinguishing acute heart failure patients survive during 1 year follow up from to those died by hemoglobin, heart rate, serum sodium and serum miR-30d level, respectively. AUC, area under the ROC curve.

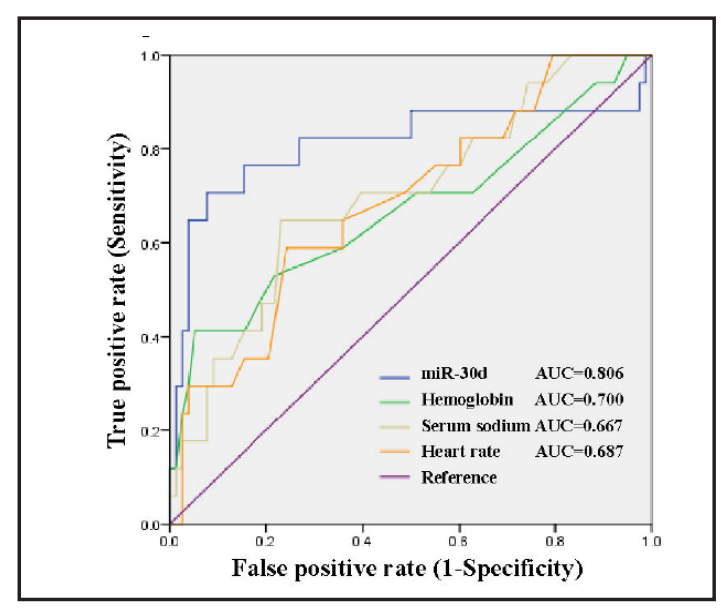

proBNP, and though the AUC area reached 0.850, no statistical difference was observed when compared to miR-30d (Fig. 3C).

\section{Serum miR-30d and mortality}

Kaplan-Meier survival analysis confirmed that patients with higher serum miR-30d levels had significantly lower mortality $(\mathrm{P}=0.001)$ (Fig. 5). Taken together, these results extend our previous studies that suggested an association between circulating miR-30d and cardiac remodeling to suggest a potential association with a clinically meaningful endpoint (mortality) in patients with AHF. 


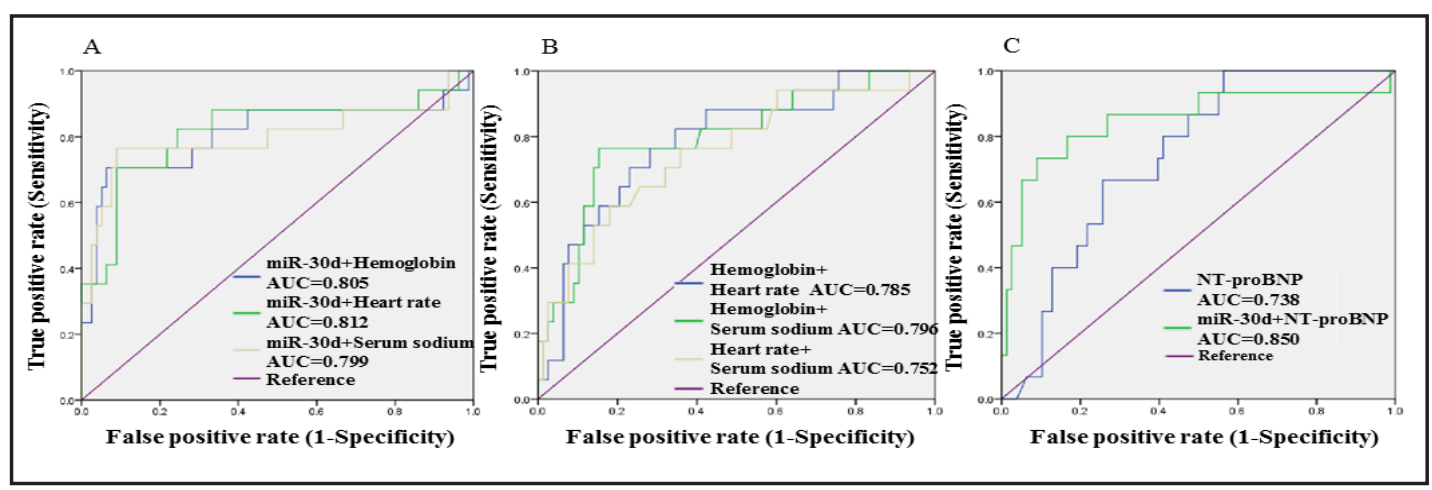

Fig. 3. The receiver-operator characteristic (ROC) curves for acute heart failure patients survive during 1 year follow up comparing to those died with two combined variables. The receiver-operator characteristic (ROC) curves for distinguishing acute heart failure patients survive during 1 year follow up from to those died by combining two variables with (A) or without (B) miR-30d and combining miR-30d with NT-pro BNP (C), respectively. AUC, area under the ROC curve.

Fig. 4. The receiver-operator characteristic (ROC) curves for acute heart failure patients survive during 1 year follow up comparing to those died with three or four combined variables. The receiver-operator characteristic (ROC) curves for distinguishing acute heart failure patients survive during 1 year follow up from to those died by hemoglobin + heart rate + serum sodium, hemoglobin+serum sodium + serum miR-30d, heart rate + serum sodium + serum miR30d,and hemoglobin +heart rate + serum sodium+ serum miR-30d, respectively. AUC, area under the ROC curve.

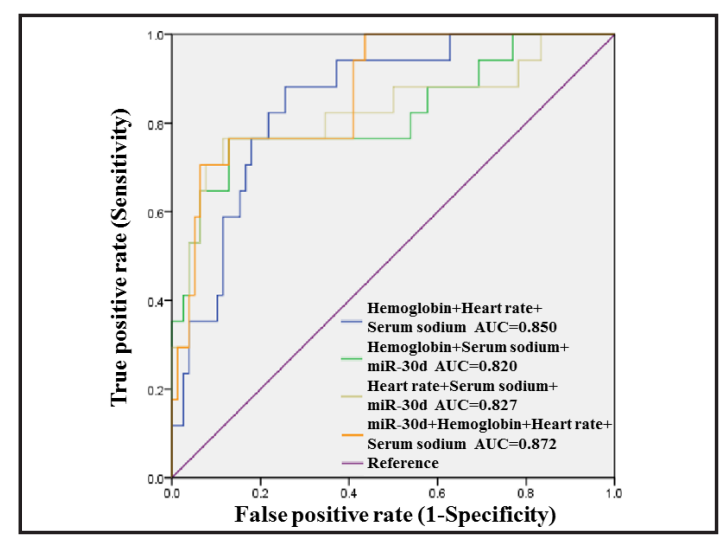

Fig. 5. Kaplan-Meier cumulative survival analysis according to serum miR-30d levels. The acute heart failure patients in this study were divided into two groups according to the median of miR-30d (lower than median vs higher than median). Survival rate free of all cause of death based on serum miR-30d level.

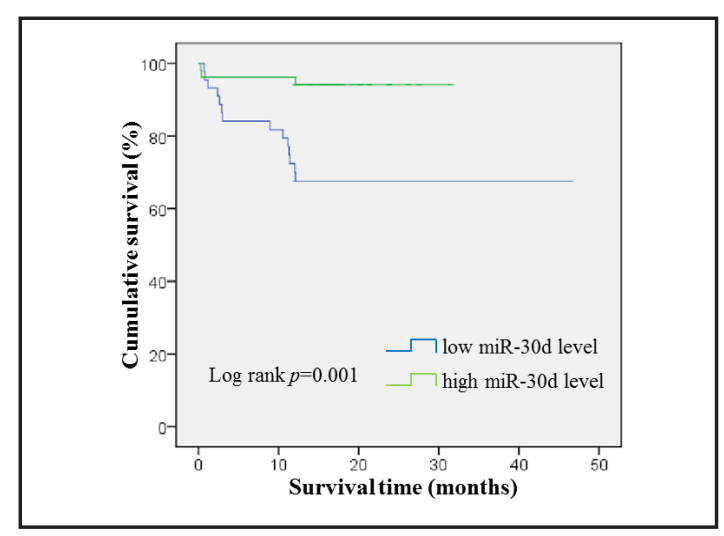

\section{Discussion}

Identification of AHF patients who are at high risk of mortality following discharge is of critical importance to guide therapeutic decision making for physicians [1]. Identification of high-risk AHF patients could facilitate allocation of resources including ventricular assist device support, cardiac transplantation, and enrollment into AHF management programs [2, 27]. Nevertheless, accurate risk assessment remains a challenging task [28]. Thus, exploring novel biomarkers for prediction of survival in AHF patients is greatly needed [27].

Several biomarkers measured during an AHF admission have been reported to independently predict death after discharge, including soluble suppression of tumorigenicity

\section{KARGER}




\section{Cellular Physiology Cell Physiol Biochem 2017;41:865-874 \begin{tabular}{ll|l} 
and Biochemistry Published online: February 16, 2017 & $\begin{array}{l}\text { (c) } 2017 \text { The Author(s). Published by S. Karger AG, Basel } \\
\text { www.karger.com/cpb }\end{array}$ \\
\hline
\end{tabular} \\ Xiao et. al.: Serum miR-30d Predicts Survival in AHF}

2 (sST2), galectin-3, copeptin, and mid-regional pro-adrenomedullin etc. [1, 29-31]. miRNAs have been found to exist in circulation in a consistent and reproducible manner, making them attractive candidates for biomarker development in AHF patients [22, 23]. Growing evidence has showed that several miRNAs can predict outcome in AHF patients, including miR-423$5 p$, miR-18a-5p, and miR-652-3p [22,23]. Recently, we have reported that circulating miR$30 \mathrm{~d}$ was associated with response to cardiac resynchronization therapy in HF [24]. miR$30 \mathrm{~d}$ was found to have a higher diagnostic value than cTnI for the early diagnosis of acute myocardial infarction (AMI), but it was not associated with 6 month cardiovascular events in AMI patients [32]. In the present study, we have found that AHF patients with higher serum miR-30d levels had significantly lower mortality even after adjustment for clinical and biochemical variable, thereby providing evidence that circulating miR-30d may be a novel prognostic biomarker for survival in AHF patients. It would be interesting to investigate how other biomarkers (sST2, galectin-3, copeptin, etc.) may relate to circulating levels of miR-30d as a novel biomarker in the future.

Our previous study reported a cardiac origin for circulating miR-30d, and cardiomyocyte as the possible source of the extracellular miR-30d [24]. In another study, miR-30d was reported to be absorbed by the failing heart [33]. We have previously shown that miR-30d acts as a 'protecteomir', with its upregulation associated with molecular features of adaptive hypertrophy, and cardioprotection against TNF- $\alpha$ mediated apoptosis [24]. Although in other models of cardiovascular diseases, miR-30d has been associated with cardiomyocyte pyroptosis [34], this study supports our findings that higher levels of circulating miR-30d are likely cardioprotective. Moreover, we also found lower incidence of atrial fibrillation in miR$30 \mathrm{~d}$ high level group and as atrial fibrillation is a major cause of cardiovascular morbidity and mortality, this relationship was in accordance with our findings. We speculated that miR-30d might also be protective for AHF in animal models. Nonetheless, definitive proof of the functional role of miR-30d can only come from gain-of-function and loss-of-function experiments in animal models. Besides that, we previously have shown that miR-30d was induced to release by increased mechanical stress [24], which might be similar in AHF patients.

Several limitations of the present study should be highlighted. Importantly, rather than performing microarrays or RNA sequencing, we only measured miR-30d based on our previous study [24]. As has been conventionally accepted, we normalized miR-30d levels to an exogenous spike-in. The issue of normalization of biofluid extracellular RNAs remains a hotly debated topic in the field as no commonly internal house-keeping control has been accepted, and we agree that this remains a limitation in the field. Nonetheless, we took care to use non-hemolyzed specimens and measured miR-30d in the same amount of sample for each patient. In addition, our sample size was limited to 96 patients, and undoubtedly our findings will need to be validated in larger studies and other populations. Besides that, the standard deviations for NT-proBNP and CK-MB are high, which is due to the heterogeneous nature of the AHF populations and the inclusion of outlier values as well. Moreover, it remains to be determined if miR-30d could response to treatment and if miR-30d guided therapy in AHF could achieve additional benefits. Finally, whether miR-30d could be a therapeutic target for AHF remains to be determined.

In conclusion, our study provides novel insights into the predictive value of circulating miR-30d for 1-year mortality in AHF patients. Large multicentre investigations to validate our findings would be highly desired to expedite the transition to clinical utilization.

\section{Acknowledgements}

This work was supported by the grants from National Natural Science Foundation of China (81570362 and 81200169 to JJ Xiao, 81370332 and 81170201 to XL Li, 81400647 to Y Bei), Shanghai Medical Guide Project from Shanghai Science and Technology Committee (14411971600 to JF Jiang), the development fund for Shanghai talents (to JJ Xiao), the Priority 


\section{Cellular Physiology Cell Physiol Biochem 2017;41:865-874

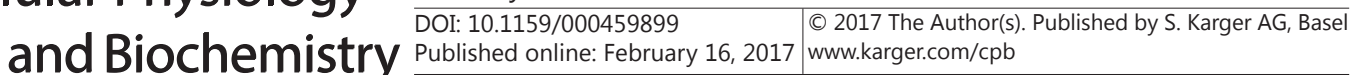 \\ Xiao et. al.: Serum miR-30d Predicts Survival in AHF}

Academic Program Development of Jiangsu Higher Education Institutions (PAPD20102013 to XL Li) and the National Institutes of Health (NCATS grant UH3 TR000901 to S Das) and Twelve-Fifth National Key Technology R\&D Program (2011BAL11B08 to XL Li). Dr XL Li is an Associate Fellow at the Collaborative Innovation Center for Cardiovascular Disease Translational Medicine and F Zhou is a Fellow at the Six Talents Peak Project of Jiangsu Province [WSN-031(IB15)].

\section{Disclosure Statement}

The authors declare there are no conflicts of interest.

\section{References}

1 Maisel AS, Choudhary R: Biomarkers in acute heart failure--state of the art. Nat Rev Cardiol 2012;9:478490.

2 Jumean MF, Kiernan MS: Determinants of survival following hospitalization for acute heart failure. Curr Heart Fail Rep 2014;11:201-211.

3 Yanavitski M, Givertz MM: Novel biomarkers in acute heart failure. Curr Heart Fail Rep 2011;8:206-211.

4 Maisel AS, Richards AM, Pascual-Figal D, Mueller C: Serial ST2 testing in hospitalized patients with acute heart failure. Am J Cardiol 2015;115:32B-37B.

5 Pascual-Figal DA, Caballero L, Sanchez-Mas J, Lax A: Prognostic markers for acute heart failure. Expert Opin Med Diagn 2013;7:379-392.

6 Givertz MM, Teerlink JR, Albert NM, Westlake Canary CA, Collins SP, Colvin-Adams M, Ezekowitz JA, Fang JC, Hernandez AF, Katz SD, Krishnamani R, Stough WG, Walsh MN, Butler J, Carson PE, Dimarco JP, Hershberger RE, Rogers JG, Spertus JA, Stevenson WG, Sweitzer NK, Tang WH, Starling RC: Acute decompensated heart failure: update on new and emerging evidence and directions for future research. J Card Fail 2013;19:371389.

$7 \quad$ Vasile VC, Jaffe AS: Emerging biomarkers for acute heart conditions. Curr Opin Cardiol 2014;29:312-318.

8 Li S, Fan Q, He S, Tang T, Liao Y, Xie J: MicroRNA-21 negatively regulates Treg cells through a TGF- $\beta 1 /$ Smadindependent pathway in patients with coronary heart disease. Cell Physiol Biochem 2015;37:866-878.

9 Shao Y, Geng Y, Gu W, Huang J, Pei H, Jiang J: Prognostic role of tissue and circulating microRNA-200c in malignant tumors: a systematic review and meta-analysis. Cell Physiol Biochem 2015;35:1188-1200.

10 Zuo K, Zhi K, Zhang X, Lu C, Wang S, Li M, He B: A dysregulated microRNA-26a/EphA2 axis impairs endothelial progenitor cell function via the p38 MAPK/VEGF pathway. Cell Physiol Biochem 2015;35:477488.

11 Liu X, Xiao J, Zhu H, Wei X, Platt C, Damilano F, Xiao C, Bezzerides V, Boström P, Che L, Zhang C, Spiegelman BM, Rosenzweig A: miR-222 is necessary for exercise-induced cardiac growth and protects against pathological cardiac remodeling. Cell Metab 2015;21:584-595.

12 Wang H, Bei Y, Shi J, Xiao J, Kong X: Non-Coding RNAs in Cardiac Aging. Cell Physiol Biochem 2015;36:16791687.

13 Xiao J, Liang D, Zhang H, Liu Y, Zhang D, Liu Y, Pan L, Chen X, Doevendans PA, Sun Y, Liang X, Sluijter JP, Chen YH: MicroRNA-204 is required for differentiation of human-derived cardiomyocyte progenitor cells. J Mol Cell Cardiol 2012;53:751-759.

14 Xiao J, Liang D, Zhang Y, Liu Y, Zhang H, Liu Y, Li L, Liang X, Sun Y, Chen YH: MicroRNA expression signature in atrial fibrillation with mitral stenosis. Physiol Genomics 2011;43:655-664.

15 Lv D, Liu J, Zhao C, Sun Q, Zhou Q, Xu J, Xiao J: Targeting microRNAs in Pathological Hypertrophy and Cardiac Failure. Mini Rev Med Chem 2015;15:475-478.

16 Wang H, Bei Y, Shen S, Huang P, Shi J, Zhang J, Sun Q, Chen Y, Yang Y, Xu T, Kong X, Xiao J: miR-21-3p controls sepsis-associated cardiac dysfunction via regulating SORBS2. J Mol Cell Cardiol 2016;94:43-53. 


\section{Cellular Physiology Cell Physiol Biochem 2017;41:865-874

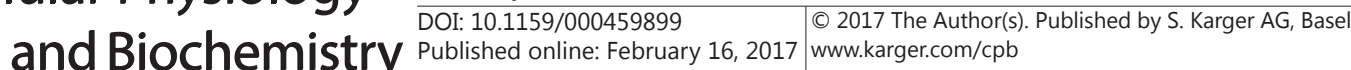 \\ Xiao et. al.: Serum miR-30d Predicts Survival in AHF}

17 Xiao J,Jing ZC, Ellinor PT, Liang D, Zhang H, Liu Y, Chen X, Pan L, Lyon R, Liu Y, Peng LY, Liang X, Sun Y, Popescu LM, Condorelli G, Chen YH: MicroRNA-134 as a potential plasma biomarker for the diagnosis of acute pulmonary embolism.J Transl Med 2011;9:159.

18 Wang KJ, Zhao X, Liu YZ, Zeng QT, Mao XB, Li SN, Zhang M, Jiang C, Zhou Y, Qian C, Feng KG, Guan HQ Tang TT, Cheng X, Chen ZJ: Circulating miR-19b-3p, miR-134-5p and miR-186-5p are promising novel biomarkers for early diagnosis of acute myocardial infarction. Cell Physiol Biochem 2016;38:1015-1029.

19 Xu J, Zhao J, Evan G, Xiao C, Cheng Y, Xiao J: Circulating microRNAs: novel biomarkers for cardiovascular diseases. J Mol Med (Berl) 2012;90:865-875.

20 Li HY, Zhao X, Liu YZ, Meng Z, Wang D, Yang F, Shi QW: Plasma microRNA-126-5p is associated with the complexity and severity of coronary artery disease in patients with stable angina pectoris. Cell Physiol Biochem 2016;39:837-846.

21 Li J, Xu J, Cheng Y, Wang F, Song Y, Xiao J: Circulating microRNAs as mirrors of acute coronary syndromes: MiRacle or quagMire? J Cell Mol Med 2013;17:1363-1370.

22 Seronde MF, Vausort M, Gayat E, Goretti E, Ng LL, Squire IB, Vodovar N, Sadoune M, Samuel JL, Thum T, Solal AC, Laribi S, Plaisance P, Wagner DR, Mebazaa A, Devaux Y; GREAT network:Circulating microRNAs and Outcome in Patients with Acute Heart Failure. PloS One 2015;10:e0142237.

23 Ovchinnikova ES, Schmitter D, Vegter EL, Ter Maaten JM, Valente MA, Liu LC, van der Harst P, Pinto YM, de Boer RA, Meyer S, Teerlink JR, O'Connor CM, Metra M, Davison BA, Bloomfield DM, Cotter G, Cleland JG, Mebazaa A, Laribi S, Givertz MM, Ponikowski P, van der Meer P, van Veldhuisen DJ, Voors AA, Berezikov E:Signature of circulating microRNAs in patienats with acute heart failure. Eur J Heart Fail 2016;18:414423.

24 Melman YF, Shah R, Danielson K, Xiao J, Simonson B, Barth A, Chakir K, Lewis GD, Lavender Z, Truong QA, Kleber A, Das R, Rosenzweig A, Wang Y, Kass DA, Singh JP, Das S: Circulating MicroRNA-30d Is Associated With Response to Cardiac Resynchronization Therapy in Heart Failure and Regulates Cardiomyocyte Apoptosis: A Translational Pilot Study. Circulation 2015;131:2202-2216.

25 Gheorghiade M, Follath F, Ponikowski P, Barsuk JH, Blair JE, Cleland JG, Dickstein K, Drazner MH, Fonarow GC, Jaarsma T, Jondeau G, Sendon JL, Mebazaa A, Metra M, Nieminen M, Pang PS, Seferovic P, Stevenson LW, van Veldhuisen DJ, Zannad F, Anker SD, Rhodes A, McMurray JJ, Filippatos G; European Society of Cardiology; European Society of Intensive Care Medicine:Assessing and grading congestion in acute heart failure: a scientific statement from the acute heart failure committee of the heart failure association of the European Society of Cardiology and endorsed by the European Society of Intensive Care Medicine. Eur J Heart Fail 2010;12:423-433.

26 Mebazaa A: Current ESC/ESICM and ACCF/AHA guidelines for the diagnosis and management of acute heart failure in adults--are there differences? Pol Arch Med Wewn 2009;119:569-573.

27 Cohen-Solal A, Laribi S, Ishihara S, Vergaro G, Baudet M, Logeart D, Mebazaa A, Gayat E, Vodovar N, PascualFigal DA, Seronde MF: Prognostic markers of acute decompensated heart failure: the emerging roles of cardiac biomarkers and prognostic scores. Arch Cardiovasc Dis 2015;108:64-74.

28 McCullough PA, Jefferies JL: Novel markers and therapies for patients with acute heart failure and renal dysfunction. Am J Med 2015;128:312 e311-e322.

29 van der Velde AR, Meijers WC, de Boer RA: Biomarkers for risk prediction in acute decompensated heart failure. Curr Heart Fail Rep 2014;11:246-259.

30 Heil B, Tang WH: Biomarkers: Their potential in the diagnosis and treatment of heart failure. Cleve Clin J Med 2015;82:S28-S35.

31 Peacock WF: Novel biomarkers in acute heart failure: MR-pro-adrenomedullin. Clin Chem Lab Med 2014;52:1433-1435.

32 Jia K, Shi P, Han X, Chen T, Tang H, Wang J:Diagnostic value of miR-30d-5p and miR-125b-5p in acute myocardial infarction. Mol Med Rep 2016;14:184-194.

33 Marques FZ, Vizi D, Khammy O, Mariani JA, Kaye DM:The transcardiac gradient of cardio-microRNAs in the failing heart. Eur J Heart Fail 2016;18:1000-1008.

34 Li X, Du N, Zhang Q Li J, Chen X, Liu X, Hu Y, Qin W, Shen N, Xu C, Fang Z, Wei Y, Wang R, Du Z, Zhang Y, Lu Y: MicroRNA-30d regulates cardiomyocyte pyroptosis by directly targeting foxo3a in diabetic cardiomyopathy. Cell Death Dis 2014;5:e1479. 